\title{
The Value of Complexity
}

\author{
A Response to Elizabeth Mowat E Brent Davis
}

\author{
PAUL CILLIERS \\ University of Stellenbosch (South Africa)
}

Interpreting Embodied Mathematics using Network Theory: Implications for Mathematics Education is a thought provoking paper, full of ideas which demand further discussion. These discussions should take place in several different contexts. In my reflection I will focus on the notion of Complexity. What is it and how does it help us to engage with the world? Does the acknowledgement that things are complex solve our problems? In this respect the paper makes a number of claims which will be looked at, starting with the more general, moving to the more specific.

The most general claim made is that the teaching of mathematics is embedded in various layers of complexity, that insights from network theory can help us to understand this better and thus enable us to perform the task of teaching mathematics more effectively. This is an important point, one with which there can be little argument. This insight is, of course, a valuable one for all forms of teaching, not just for mathematics

This general claim is based on the argument that mathematics itself is a complex system. This claim is much less self-evident, and needs carefully scrutiny. My knowledge of mathematics is too limited to perform this task exhaustively, but some critical questions may open up the discussion.

The first question which jumps to mind concerns the exact range of the notion "mathematics". If "embodied mathematics" refers to the way in which individual human agents understand mathematics, there is no big problem. Our understanding is certainly a complex thing, but then, our understanding is a complex thing in general, not 
only where mathematics is concerned. If, however, "mathematics" refers to the whole subject or discipline, it becomes rather difficult to understand what the "system" of mathematics could be. There are so many different kinds of mathematics. What is more, mathematics is normally considered to be "formal". A self-consistent formal system can hardly be described as complex. Of course there are incomplete formal systems, but does incompleteness indicate complexity, or simply paradox? And even if this is so, how much of "mathematics" is Gödelian? The largest part of mathematics normally used can be given a purely formal description, and I would hesitate to call it complex.

The authors base their claim that mathematics is a complex system on the fact that it can be described in terms of the ten characteristics I use in Complexity and Postmodernism (Cilliers 1998). On this level their argument is fairly convincing and can certainly generate meaningful insights. Nevertheless, pace myself, is it enough to simply find these ten characteristics in a somewhat direct way in order to claim complexity? It is clear to me now, thanks to the reflection triggered by the paper under discussion, that more is required. These characteristics only form part of the process of interpretation necessary to acknowledge complexity. At least two elements crucial to complexity are absent from the discussion.

The first concerns the central importance of memory in complex systems (the word "memory" is not used in the paper). In what sense does mathematics have a memory, especially if one keeps in mind that the memory of a complex system is contingent and dynamic? Perhaps an argument for "memory in mathematics" can be made, but it needs a much more elaborate discussion than the brief remarks made when discussing characteristic number nine (complex systems have a history).

A second, and even more problematic question concerns the notion of emergence. One of the most defining characteristics of complex systems is that they have emergent properties, i.e. properties which cannot simply be reduced to properties of components in the system. To what extent does mathematics have emergent properties? There are clearly forms of emergence in the teaching of mathematics -understanding itself can be seen as a form of emergence - but in mathematics itself? This seems to me like an extremely interesting question, but it is not addressed at all.

The point I am trying to make is not that mathematics is not complex, but that we need a more detailed argument for it, an argument which should be informed by a deeper engagement with Complexity than just referring to Cilliers' ten Characteristics. Someone who did do battle with these issues extensively is Robert Rosen (1991). His ideas on the use of category theory to model complex organizations in mathematics and in natural systems, and later extensions of this branch of mathematics (Awodey, 2006), are indispensable to a discussion of the complexity of mathematics.

The issues discussed up to now are concerned with a number of detailed matters within complexity theory. A larger, overarching question remains: Does complexity theory advance our understanding of mathematics and its pedagogy beyond the acknowledgement that knowledge is embodied and that metaphors play an important role in the process of generating understanding? One could of course arrive at these insights without invoking complexity. I would argue that the acknowledgement of 
complexity is crucial, but to make the argument stick, a much more detailed engagement with a variety of ideas in Complexity is required. Again, there are two important components to this engagement.

In the first place one has to acknowledge that the "discipline" of Complexity is a house divided. There are serious differences between different approaches to complexity. After about two or three decades of work explicitly dedicated to the understanding of complex systems, it has become crucial to reflect critically on the value of these different approaches. One way of distinguishing between these approaches is provided by Edgar Morin (2007) who distinguishes between "general" and "restricted" complexity. Restricted complexity refers mainly to the mathematical and computational approaches to complexity, often strongly informed by chaos theory. This approach, Morin argues, acknowledges the non-linear, relational nature of complex systems, but seeks to tame it in ways which reintroduces positivism and reductionism. General complexity on the other hand, argues for the limits of all approaches to complex systems and urges that we acknowledge these limits and recognise that we need a new language in which to do this, a language which moves beyond Enlightenment ideals of neutrality and objectivity. Whether we can find such a language remains, also for Morin, a contested idea.

The argument is not that restricted complexity is wrong and general complexity correct. The argument is that these approaches are different, and that we have to acknowledge the different values they embody. When we have to solve a specific problem in the real world we cannot involve life, the universe and everything. We have to frame the problem in a specific way and use specific tools and methods. This process is restricted by necessity. Nevertheless, this does not lead to a final, complete and objective understanding of the complex issues at hand. Our solutions and our understanding remains provisional. We will only take this provisionality seriously if we constantly return to the critical reflection necessitated by a "general" understanding of the complex world we live in.

The upshot of this acknowledgement leads us to the second component vital to a responsible engagement with complexity. If there is no neutral or objective framework for understanding complexity, it implies that we have to make choices which cannot be reduced to calculation alone. An acknowledgement of complexity leads to the acknowledgment of the inevitable role played by values (see Cilliers 2005). It is surprising that the words "value", "ethics" or "normativity" do not feature in the paper at all. If we wish to find ways of understanding a complex world better, and to find ways in which to teach this understanding, then an engagement with the "ethics of complexity" will have to be a priority.

Acknowledgement

This response has been informed by discussions with Jannie Hofmeyr, co-director of the Centre for Studies in Complexity at Stellenbosch University. 


\section{About the Author}

Paul Cilliers is professor of Complexity and Philosophy at the University of Stellenbosch in South Africa, where he co-directs the Centre for Studies in Complexity. His research is focused on the philosophical and ethical implications of complexity theory and he has published widely in the field.

\section{References}

Awodey, S. (2006) Category theory. Oxford: Clarendon Press.

Cilliers, P. (1998). Complexity and postmodernism. Understanding complex systems. London: Routledge. Cilliers, P. (2005). Complexity, deconstruction and relativism. Theory Culture \& Society, 22(5): 255-267. Morin, E. (2007). Restricted complexity, general complexity, in: C. Gershenson, D. Aerts \& B. Edmonds (Eds), Worldviews, science and us: Philosophy and complexity. Singapore: World Scientific. 5-29.

Rosen, R. (1991) Life Itself: A comprehensive inquiry into the nature, origin, and fabrication of life. New York: Columbia University Press.

(C) Copyright 2010. The author, PAUL CILLIERS assigns to the University of Alberta and other educational and non-profit institutions a non-exclusive license to use this document for personal use and in courses of instruction provided that the article is used in full and this copyright statement is reproduced. The author also grants a non-exclusive license to the University of Alberta to publish this document in full on the World Wide Web, and for the document to be published on mirrors on the World Wide Web. Any other usage is prohibited without the express permission of the authors. 